\title{
ASSESSMENT OF SIMULATED COMPACT POLARIMETRY OF THE RCM MEDIUM RESOLUTION SAR MODES FOR OIL SPILL DETECTION
}

\author{
Mohammed Dabboor ${ }^{1}$, Suman Singha ${ }^{2}$, Benoit Montpetit $^{3}$, Benjamin Deschamps ${ }^{4}$, Dean Flett ${ }^{4}$ \\ ${ }^{1}$ Meteorological Research Division, Environment and Climate Change Canada, Canada \\ ${ }^{2}$ Remote Sensing Technology Institute (IMF), German Aerospace Center (DLR), Germany \\ ${ }^{3}$ Wildlife and Landscape Science Division, Environment and Climate Change Canada, Canada \\ ${ }^{4}$ Canadian Ice Service, Environment and Climate Change Canada, Canada
}

\begin{abstract}
Operational detection and discrimination of oil spills over oceans has received considerable attention due to its impact on marine ecosystem from environmental and political points of view. Synthetic Aperture Radar (SAR) is a valuable instrument for maritime pollution monitoring. The three main requirements for effective operational oil spill detection using SAR are: 1) low noise floor, 2) large area coverage, and 3) maximizing detection and discrimination of pollution and 'lookalike' features, by polarization diversity, multiple frequency, etc. In order to reconcile the advantages of fully polarimetric SAR with larger area coverage, compact polarimetry (CP) acquisitions offer a trade-off between the above mentioned requirements. The future Canadian RADARSAT Constellation Mission (RCM) will enable the acquisition of CP SAR data in wide swath imagery, including ScanSAR modes. In this study, we investigate the potential of CP from three RCM SAR modes for oil spill detection. Results indicate that the RCM MR30 SAR mode has promising oil spill detection performance.
\end{abstract} spill.

Index Terms - RCM, compact polarimetric SAR, oil

\section{INTRODUCTION}

Spaceborne SAR has attracted significant research interest for maritime pollution monitoring [1]. A major focus is on oil spill detection near offshore platforms. As most of the offshore platforms reside in environmentally sensitive zones and also near coasts, they pose a serious threat to marine and coastal ecosystems in long term. Currently, most of the operational oil spill monitoring systems uses singlepolarization (mainly VV) intensity SAR images where oil spills appear as dark slicks [1]. Although spaceborne SAR has proven to be a valuable tool for oil spill detection,

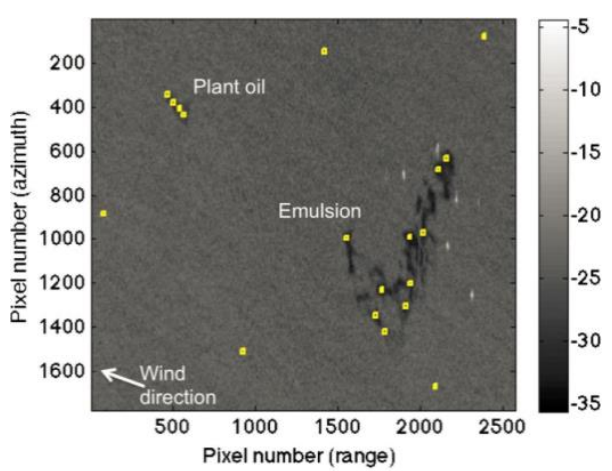

Fig. 1. The test site with emulsion and plant oil [4].

challenges still remain, such as the discrimination between oil and 'lookalike' features.

The RCM is a future Canadian SAR mission, to be launched in late 2018, which will provide compact polarimetry as a polarization option for all non-quadpolarization imaging modes [2,3]. A CP SAR system operates with half pulse repetition frequency, reducing the average transmitted power and increasing the swath width [3]. The wider coverage of the CP SAR system reduces the revisit time of the satellite, making this system operationally viable, especially for applications such as maritime pollution [3]. In this study, we investigate the potential of CP from three RCM SAR modes for oil spill detection. Herein, we assess all the RCM medium resolution SAR modes. These modes are: 1) the Medium Resolution 50m (MR50), 2) the Medium Resolution 30m (MR30), and 3) the Medium Resolution 16m (MR16). Table I presents the characteristics of each of the tested SAR modes. CP parameters are simulated for each of the tested RCM SAR modes and investigated for oil spill detection. The most effective CP parameters for oil spill detection in each mode are extracted and used for classification of oil spills and lookalike using the Random Forest (RF) classification algorithm.

\section{DATA AND TEST SITE}


The test site for this study is located in the North Sea offshore Norway. A large-scale oil spill exercise at the Frigg field was conducted in June 2011 and previously reported in [4]. Crude Oil (CO), Emulsion (EM) which is mixture of oil and water, and plant oil to be treated as lookalike (LA) were spilled during the experiment. Oil spills were captured using two RADARSAT-2 fine quad-pol SAR images with incidence angles equal to $46.7^{\circ}$ and $35.3^{\circ}$ for the first and the second RADARSAT-2 image, respectively. Details of the properties of the spilled oil samples, weather conditions and ancillary information can be found in [4-5]. Fig. 1 shows the first RADARSAT-2 image (VV) containing EM and LA [4]. For the purpose of the abstract, we present the results of the first RADARSAT-2 image, which contains EM and LA only (Figure 1). Also, similar to the adopted strategy in [4], we masked out the water in the first image and focused the study on the discrimination between EM and LA.

\section{DATA PROCESSING}

A RCM data simulator, developed at the Canada Center for Mapping and Earth Observation, was used to simulate RCM CP SAR data. The simulator calculates and generates $23 \mathrm{CP}$ SAR parameters for each mode. Table II presents the extracted CP parameters by the RCM simulator. Details on the derived CP SAR parameters and their calculation can be found in [6]. Samples of oil spill and LA were collected and used for separability estimation in each CP parameter using the Kolmogorov-Smirnov (K-S) nonparametric distance, which takes values between 0 and 1 . Thus, CP parameters with discrimination capabilities between oil spills and LA were identified. The correlation between the identified CP parameters was also analyzed in a subsequent processing step by estimating the Spearman correlation coefficient between parameters. The purpose of this analysis was the extraction of effective less-correlated CP parameters for the oil spill classification using the RF classification algorithm.

\section{RESULTS}

Fig. 2 shows the calculated K-S distance between EM and LA for the RCM MR16 (Fig. 2a), MR30 (Fig. 2b), and MR50 (Fig. 2c) SAR modes. Assuming that a CP parameter with discrimination capability between EM and LA should have a K-S distance between EM and LA > 0.5, we note that only one CP parameter $\left(\sigma_{\mathrm{RL}}^{0}\right)$ can separate between EM and LA in the MR16 mode with K-S distance equal to 0.58 . In the MR30 mode, the number of parameters increases to four with the $\sigma_{\mathrm{RL}}^{0}$ backscattering coefficient to provide the highest K-S distance between EM and LA (0.74). The lowest distance is given by both the SE_Int and SV0 parameters (0.69). The number of $\mathrm{CP}^{-}$parameters showing discrimination capability between EM and LA becomes two
Table I

Characteristics of Tested RCM SAR Modes

\begin{tabular}{|c|c|c|c|c|}
\hline $\begin{array}{c}\text { Beam } \\
\text { Mode }\end{array}$ & $\begin{array}{c}\text { Nom. Res. } \\
(\mathrm{m})\end{array}$ & $\begin{array}{c}\text { Swath } \\
(\mathrm{km})\end{array}$ & $\begin{array}{c}\text { Looks } \\
(\mathrm{rng} \mathrm{x} \mathrm{az})\end{array}$ & $\begin{array}{c}\text { Noise Floor } \\
(\mathrm{dB})\end{array}$ \\
\hline MR50 & 50 & 350 & $4 \mathrm{x} 1$ & -22 \\
\hline MR30 & 30 & 125 & $2 \times 2$ & -24 \\
\hline MR16 & 16 & 30 & $1 \times 4$ & -25 \\
\hline
\end{tabular}

Table II

Simulated RCM CP Parameters

\begin{tabular}{|c|c|}
\hline Short form & Description \\
\hline $\begin{array}{l}\text { SV0, SV1, SV2, } \\
\text { SV3 }\end{array}$ & Stokes vector elements \\
\hline SE_Pol, SE_Int & $\begin{array}{l}\text { Shannon entropy polarimetric and } \\
\text { intensity components }\end{array}$ \\
\hline$\sigma_{\mathrm{RL}}^{0}, \sigma_{\mathrm{RR}}^{0}, \sigma_{\mathrm{RH}}^{0}, \sigma_{\mathrm{RV}}^{0}$ & $\begin{array}{l}\text { Sigma naught backscattering - right } \\
\text { circular transmit and left circular, right } \\
\text { circular, linear horizontal or linear } \\
\text { vertical receive polarization }\end{array}$ \\
\hline$\sigma_{\mathrm{RV}}^{0} / \sigma_{\mathrm{RH}}^{0}$ & Right co-polarized ratio \\
\hline$\rho_{\text {RHRV }}$ & RH RV correlation coefficient \\
\hline $\begin{array}{c}\mathrm{m}-\delta_{-} \mathrm{S}, \mathrm{m}-\delta_{-} \mathrm{V}, \mathrm{m}- \\
\delta \_\mathrm{DB}\end{array}$ & $\begin{array}{l}\text { Surface, volume, and double bounce } \\
\text { scattering from } \mathrm{m}-\delta \text { decomposition }\end{array}$ \\
\hline $\begin{array}{c}\mathrm{m}-\chi_{\_} \text {odd }, \mathrm{m}-\chi_{\ell} \mathrm{V} \\
\mathrm{m}-\chi_{\text {_even }}\end{array}$ & $\begin{array}{l}\text { odd, volume, and even bounce scattering } \\
\text { from } \mathrm{m}-\chi \text { decomposition }\end{array}$ \\
\hline $\mathrm{m}$ & Degree of polarization \\
\hline$\delta_{\text {RHRV }}$ & RH RV phase difference \\
\hline $\mathrm{u}$ & Conformity coefficient \\
\hline$\sigma_{\mathrm{RR}}^{0} / \sigma_{\mathrm{RL}}^{0}$ & Circular polarization ratio \\
\hline$\alpha_{\mathrm{s}}$ & $\begin{array}{c}\text { Alpha parameter related to the ellipticity } \\
\text { of the compact scattered wave }\end{array}$ \\
\hline
\end{tabular}

in the RCM MR50 SAR mode, with a K-S distance equal to 0.51 for the SE_Int and SV0 parameters.

Since the MR16 SAR mode has only one CP parameter with discrimination capability between EM and LA, the Spearman correlation analysis was conducted on the MR30 and MR50 modes. We assumed two CP parameters to be strongly correlated if their estimated absolute correlation value $\mathrm{R} \geq 0.9$. Based on this assumption, $\mathrm{CP}$ parameters with discrimination capability between EM and LA were placed into groups of strongly correlated $(\mathrm{R} \geq 0.9)$ and independent (less correlated with $\mathrm{R}<0.9$ ) $\mathrm{CP}$ parameters. One representative parameter from each group of strongly correlated parameters was selected and a subset of effective $\mathrm{CP}$ parameters was formed containing the selected representative parameters and the independent. For the selection to be optimum, we chose as a representative parameter the one with the highest K-S distance between 


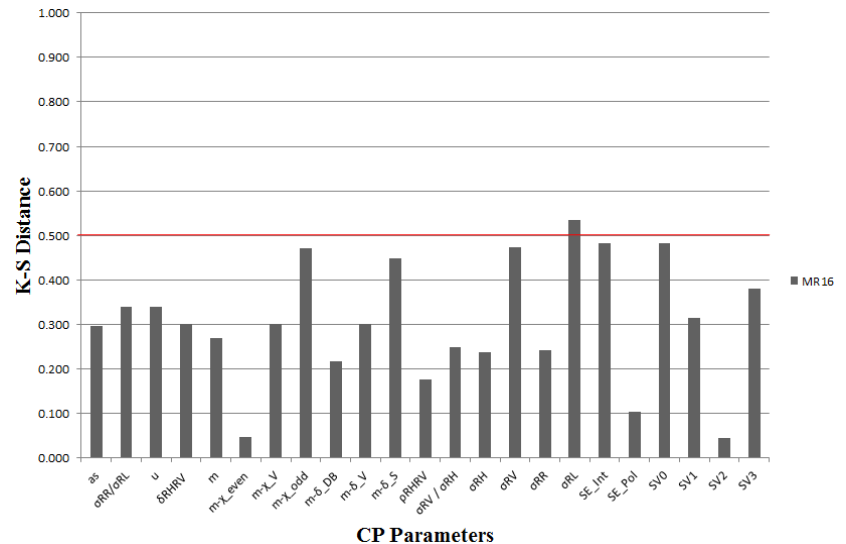

(a)

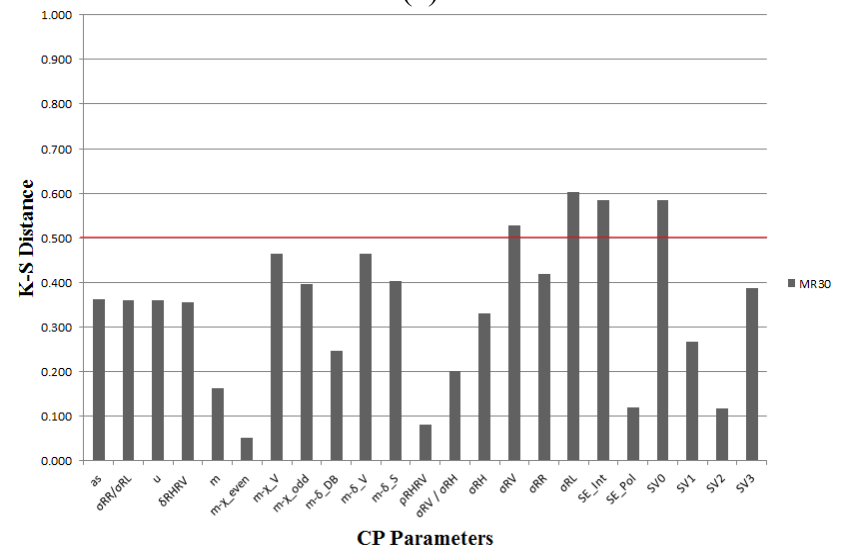

(b)

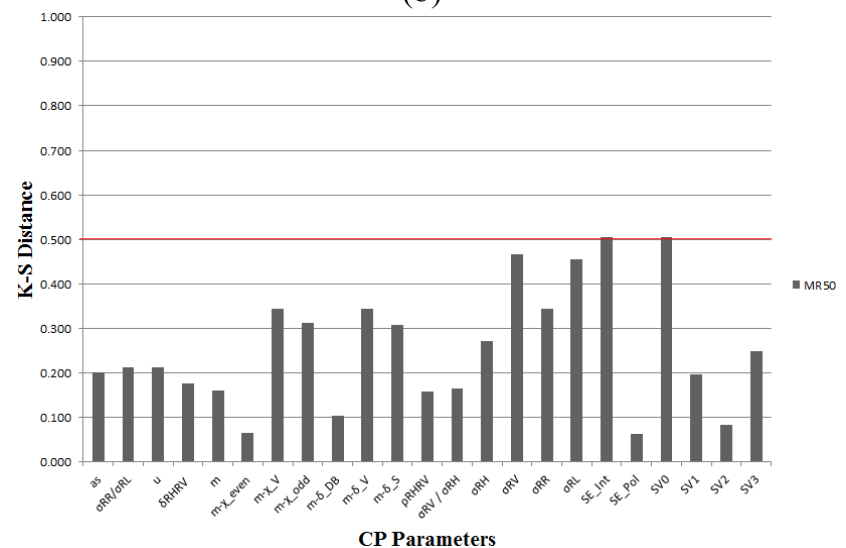

(c)

Fig. 2. K-S distance between EM and LA for the RCM a) MR16, b) MR30, and c) MR50 SAR modes.

EM and LA. Table III shows the extracted subset of each mode. Also, Fig. 3a-c show the classification results of EM and LA of each mode based on the CP parameters of each subset using the RF classification algorithm. The training of the RF algorithm was performed using half of the selected EM and LA samples, while the other half was used to estimate the classification accuracies shown in Table III. We
Table III

Effective CP Parameters of Each RCM SAR Mode and the Corresponding Classification Accuracy

\begin{tabular}{|c|c|c|c|c|c|c|}
\hline \multicolumn{2}{|c|}{} & \multicolumn{5}{|c|}{ Classification (\%) } \\
\hline Mode & CP Parameters & & LA & EM & Overall & Kappa \\
\hline \multirow{2}{*}{ MR16 } & \multirow{2}{*}{$\sigma_{\mathrm{RL}}^{0}$} & LA & 69.88 & 23.75 & 74.30 & 0.433 \\
\cline { 3 - 7 } & & EM & 29.98 & 76.25 & & \\
\hline \multirow{2}{*}{ MR30 } & $\sigma_{\mathrm{RL}}^{0}$ & LA & 62.88 & 6.66 & 84.05 & 0.599 \\
\cline { 3 - 7 } & $\sigma_{\mathrm{RV}}^{0}$ & EM & 37.12 & 93.34 & & \\
\hline \multirow{2}{*}{ MR50 } & \multirow{2}{*}{ SE_Int or SV0 } & LA & 50.38 & 1.66 & 83.70 & 0.559 \\
\cline { 3 - 7 } & & EM & 49.62 & 98.34 & & \\
\hline
\end{tabular}

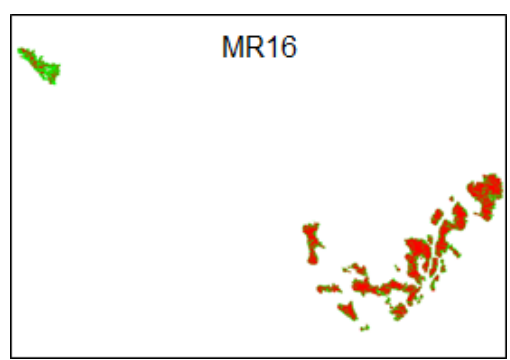

(a)

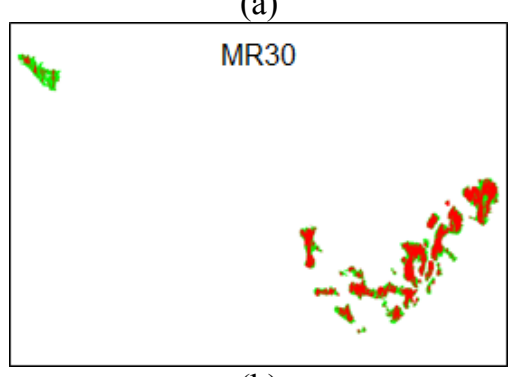

(b)

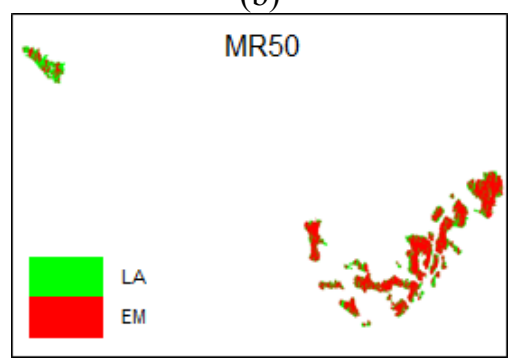

(c)

Fig. 3. Classification of EM and LA in the RCM a) MR16, b) MR30, and c) MR50 SAR modes.

masked out the open water area since the focus of the study is on the classification of EM and LA.

As shown in Table III, the RCM MR30 SAR mode had two effective less-correlated CP parameters $\left(\sigma_{\mathrm{RL}}^{0}\right.$ and $\left.\sigma_{\mathrm{RV}}^{0}\right)$, while the other two RCM modes (MR16 and MR50) had only one effective CP parameter (correlation between the SE_Int or SV0 parameters is equal to 1 ). These two parameters gave the best classification results (Fig. 3b) in comparison to the classification results of the MR16 (Fig. 3a) and MR50 (Fig. 
3c) modes. This is confirmed in Table III where the MR30 mode gives the highest overall classification accuracy $(84.05 \%)$. The lowest overall classification accuracy is given by the MR16 mode ( $74.30 \%)$.

\section{CONCLUSIONS}

A study on the potential of simulated RCM CP SAR data for oil spill detection is conducted. The study is based on $23 \mathrm{CP}$ parameters which are simulated for three medium resolution RCM SAR modes. A separability estimation of EM and LA in each CP parameter of each tested RCM SAR mode followed by a correlation analysis of these parameters led to the identification of subsets of effective $\mathrm{CP}$ parameters suitable for discrimination between EM and LA. The identified subset of parameters of each mode was used for the classification of EM and LA. Results indicate promising performance of the RCM MR30 SAR mode for oil spill detection. Results of applying the aforementioned methodology on the second RADARSAT-2 image containing CO, EM and LA will be provided.

\section{REFERENCES}

[1] S. Singha, M. Vespe, and O. Trieschmann, "Automatic Synthetic Aperture Radar Based Oil Spill Detection and Performance Estimation via a Semi-Automatic Operational Service Benchmark," Marine Pollution Bulletin, Vol.73, No. 1, pp. 199209, August 2013.

[2] G. Séguin and S. Ahmed, "RADARSAT Constellation, Project Objectives and Status," Proceeding of the IEEE International Geoscience and Remote Sensing Symposium, Cape Town, South Africa, pp II-894-II-897, 12-17 July 2009.

[3] F. Charbonneau, B. Brian, K. Raney, H. McNairn, C. Liu, P. Vachon, J. Shang, R. De Abreu, C. Champagne, A. Merzouki, T. Geldsetzer, "Compact Polarimetry Overview and Applications Assessment," Canadian Journal of Remote Sensing, Vol. 36, No. 2, p.p. S298-S315, 2014.

[4] S. Skrunes, C. Brekke, and T. Eltoft, "Characterization of Marine Surface Slicks by RADARSAT-2 Multipolarization Features," IEEE Transactions on Geoscience and Remote Sensing, Vol. 52, No. 9, pp. 5302-5319, November 2013.

[5] S. Skrunes, C. Brekke, T. Eltoft, and Vladimir Kudryavtsev, "Comparing Near-Coincident C-and X-Band SAR Acquisitions of Marine Oil Spills," IEEE Transactions on Geoscience and Remote Sensing, Vol. 53, No. 9, pp. 1958-1975, September 2014.

[6] M. Dabboor and T. Geldsetzer, "Towards Sea Ice Classification Using Simulated RADARSAT Constellation Mission Compact Polarimetric SAR Imagery," Remote Sensing of Environment, Vol. 140, pp. 189-195, January 2014. 